Available online at www.isr-publications.com/jmcs

J. Math. Computer Sci. 16 (2016), 435-444

Research Article

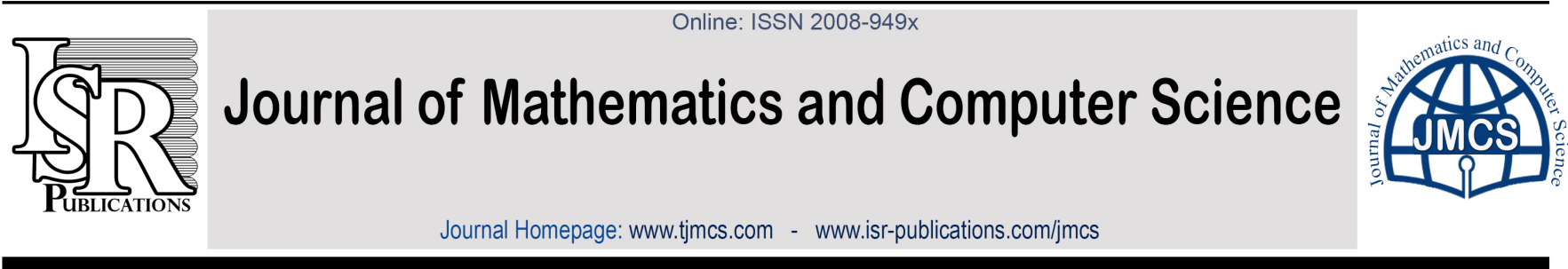

\title{
New results in quasi cone metric spaces
}

Taja Yayinga, Bipan Hazarikab,*, Huseyin Çakallic

${ }^{a}$ Department of Mathematics, Dera Natung Govt. College, Itanagar-791 009, Arunachal Pradesh, India.

${ }^{b}$ Department of Mathematics, Rajiv Gandhi University, Rono Hills, Doimukh-791 112, Arunachal Pradesh, India.

${ }^{c}$ Faculty of Arts and Sciences, Maltepe University, Marmara Ĕgltim Köyü, TR 34857, Maltepe, İstanbul-Turkey.

\begin{abstract}
In this paper, we prove some interesting results using forward and backward convergence in quasi cone metric spaces. We study forward and backward sequential compactness, sequential countably compactness, and sequential continuity property in quasi cone metric spaces and give some interesting results. (C)2016 all rights reserved.
\end{abstract}

Keywords: Quasi cone metric space, forward convergence, backward convergence, $f f$-continuous, forward complete, forward sequential countably compactness.

2010 MSC: 40A35, 40A05, 26A15.

\section{Introduction and preliminaries}

The concept of a cone metric which has been known since the middle of 20th century (see [17, 22, [27]) was reintroduced by Huang and Zhang in [14]. This concept is an interesting generalization of usual metric space where the set of real numbers is replaced by an ordered Banach space. After the study of this space by Huang and Zhang [14], a lot of work have been done on this structure. Cone metric spaces are one of the many generalizations of metric spaces which play an important role in fixed point theory, computer science, and some other research areas as well in general topology (see, for example, [1, 2, 7-11, 13, 21, 26]). There have been a lot of papers dealing with the theory of cone metric spaces. Recently, the paracompactness and the metrizability of cone metric spaces have been discussed in [15] and [24], respectively.

\footnotetext{
*Corresponding author

Email addresses: tajayaying20@gmail.com (Taja Yaying), bh_rgu@yahoo.co.in (Bipan Hazarika), huseyincakalli@maltepe.edu.tr (Huseyin Çakalli)
} 
A quasi metric is a distance function in which the symmetry axiom is eliminated in the definition of a metric (see [4, 12, 16, 18, 28, 29]). Many authors have given different definitions of quasi cone metric, see for instance [3, 25. A lot of researches have been done on quasi cone metric space since then, specially on fixed point theory. In this paper, we focus on a different direction and study two sided convergence and completeness in a quasi cone metric space. We use the definition given by Abdeljawad and Karapinar in [3]. However, it should be noted that due to the absence of real numbers (which is replaced by an ordered Banach space), the methods of proofs are not always analogous to the usual metric case. Now we recall some notions, definitions and results which will be used in this work.

Definition 1.1. A subset $P$ of a real Banach space $E$ is called a cone if

(1) $P$ is closed, nonempty, and $P \neq\{\theta\}$;

(2) if $a, b \in \mathbb{R}^{+}$and $x, y \in P$, then $a x+b y \in P$;

(3) if both $x \in P$ and $-x \in P$ then $x=\theta$.

Given a cone $P$ in $E$, we define a partial ordering $\leq$ on $E$ with respect to $P$ by $x \leq y$ if and only if $y-x \in P$. We shall write $x<y$ to indicate that $x \leq y$ but $x \neq y$, while $x \ll y$ will stand for $y-x \in \stackrel{\circ}{P}$ where $\stackrel{\circ}{P}$ denotes the interior of $P$.

In the following we always suppose that $E$ is a Banach space, $P$ is a solid cone, i.e., $\stackrel{\circ}{P} \neq \phi$ in $E$ and $\leq$ is a partial ordering with respect to $P$.

Definition $1.2([14])$. A cone metric space is an ordered pair $(X, d)$, where $X$ is a non empty set and $d: X \times X \rightarrow E$ is a mapping satisfying:

(i) $\theta \preceq d(x, y)$ for all $x, y \in X$;

(ii) $d(x, y)=\theta$ if and only if $x=y$;

(iii) $d(x, y)=d(y, x)$ for all $x, y \in X$;

(iv) $d(x, y) \preceq d(x, z)+d(z, y)$ for all $x, y, z \in X$.

Definition $1.3([3])$. Let $X$ be a nonempty set. Suppose that the mapping $d: X \times X \rightarrow E$ satisfies

(i) $\theta \preceq d(x, y)$ for all $x, y \in X$;

(ii) $d(x, y)=\theta$ if and only if $x=y$;

(iii) $d(x, y) \preceq d(x, z)+d(z, y)$ for all $x, y, z \in X$,

then $d$ is called quasi cone metric on $X$, and the pair $(X, d)$ is called quasi cone metric space.

Example 1.4. Let $X=\mathbb{R}, E=\mathbb{R}^{2}, P=\{(x, y) \in E: x, y \geq 0\}$ and $q: X \times X \rightarrow E$ defined by

$$
q(x, y)= \begin{cases}(x-y, \alpha(x-y)), & x \geq y \\ (\alpha, 1), & x<y\end{cases}
$$

then $q$ is a quasi cone metric on $X$ and $(X, q)$ is a quasi cone metric space. But it is not a cone metric space.

Definition $1.5([23])$. A sequence $\left(x_{n}\right)_{n \in \mathbb{N}}$ forward converges (resp. backward converges) to $x_{0} \in X$ if for every $c \in E$ with $\theta \ll c$ (i.e., $c-\theta \in \stackrel{\circ}{P})$ there exists a natural number $N$ such that $d\left(x_{0}, x_{n}\right) \ll c$ (resp. $d\left(x_{n}, x_{0}\right) \ll c$ ) for all $n \geq N$.

We denote this by writing $x_{n} \stackrel{f}{\rightarrow} x_{0}$ (resp. $x_{n} \stackrel{b}{\rightarrow} x_{0}$ ). 
Definition 1.6. Let $\left(X, d_{X}\right)$ and $\left(Y, d_{Y}\right)$ be two quasi cone metric spaces. A function $f: X \rightarrow Y$ is $f f-$ continuous at $x \in X$ if whenever $x_{n} \stackrel{f}{\rightarrow} x$ in $\left(X, d_{X}\right)$ we have $f\left(x_{n}\right) \stackrel{f}{\rightarrow} f(x)$ in $\left(Y, d_{Y}\right)$.

An analogous statements can be written for the other three types of continuity, namely, $f b$ continuity, $b b$-continuity, and $b f$-continuity.

Definition $1.7([23])$. Let $(X, d)$ be a quasi cone metric space. We say that a sequence $\left(x_{k}\right)_{k \in \mathbb{N}}$ is

1. forward Cauchy if for every $c \in E$ with $\theta \ll c$ there exists $N \in \mathbb{N}$ such that for $m \geq n \geq N$, $d\left(x_{n}, x_{m}\right) \ll c$ holds;

2. backward Cauchy if for every $c \in E$ with $\theta \ll c$ there exists $N \in \mathbb{N}$ such that for $m \geq n \geq N$, $d\left(x_{m}, x_{n}\right) \ll c$ holds.

Shadad and Noorani used left (right) Cauchy for forward (backward) Cauchy in [23].

Definition 1.8. A set $S \subset X$ is forward (resp. backward) sequentially compact if every sequence in $X$ has a forward (resp. backward) convergent subsequence with limit in $S$.

Definition $1.9([23])$. A set $S \subset X$ is forward (resp. backward) complete if every forward (resp. backward) Cauchy sequence is forward (resp. backward) convergent.

Lemma $1.10([20])$.

(1) If $u \leq v$ and $v \ll w$, then $u \ll w$.

(2) If $u \ll v$ and $v \leq w$, then $u \ll w$.

(3) If $u \ll v$ and $v \ll w$, then $u \ll w$.

(4) If $\theta \leq u \ll c$ for each $c \in \stackrel{\circ}{P}$ then $u=\theta$.

\section{Main results}

Lemma 2.1. Let $(X, d)$ be a quasi cone metric space. Then $x_{n} \stackrel{f}{\rightarrow} x$ if and only if each subsequence of it, is forward convergent to $x$.

Proof. Let $x_{n} \stackrel{f}{\rightarrow} x$. Then for $c \in E$ with $\theta \ll c$ there exists an $N \in \mathbb{N}$ such that $c-d\left(x, x_{n}\right) \in \stackrel{\circ}{P}$ for all $n \geq N$. Let $\left(x_{n_{k}}\right)_{k \in \mathbb{N}}$ be an arbitrary subsequence of $\left(x_{n}\right)$. If $n_{k} \geq N$, we have $c-d\left(x, x_{n_{k}}\right) \in \stackrel{\circ}{P}$, i.e., $d\left(x, x_{n_{k}}\right) \ll c$. So $x_{n_{k}} \stackrel{f}{\rightarrow} x$.

The proof of the converse is straightforward so is omitted. Hence the proof is completed.

Lemma 2.2. If $\left(x_{k}\right)_{k \in \mathbb{N}}$ forward converges to $x_{0} \in X$ and backward converges to $y_{0} \in X$ then $x_{0}=y_{0}$.

Proof. By the assumption, $x_{k} \stackrel{f}{\rightarrow} x_{0}$. Thus for $c \in E$ with $\theta \ll c$ there exists a natural number $N_{1}$ such that $d\left(x_{0}, x_{k}\right) \ll \frac{c}{2}$ for all $k \geq N_{1}$. Also $x_{k} \stackrel{b}{\rightarrow} y_{0}$, so there exists a natural number $N_{2}$ such that $d\left(x_{k}, y_{0}\right) \ll \frac{c}{2}$ for all $k \geq N_{2}$.

Let $N=\max \left\{N_{1}, N_{2}\right\}$. Then for all $k \geq N$ and $c \in E$ with $\theta \ll c, d\left(x_{0}, y_{0}\right) \leq d\left(x_{0}, x_{k}\right)+$ $d\left(x_{k}, y_{0}\right) \ll \frac{c}{2}+\frac{c}{2}=c$. This implies that $d\left(x_{0}, y_{0}\right) \ll c$ for every $c$, and so $d\left(x_{0}, y_{0}\right)=\theta$ by (4) of Lemma 1.10. Hence $x_{0}=y_{0}$.

Theorem 2.3. Let $d: X \times X \rightarrow E$ be a quasi cone metric. If $(X, d)$ is forward sequentially compact and $x_{n} \stackrel{b}{\rightarrow} x$, then $x_{n} \stackrel{f}{\rightarrow} x$. 
Proof. Let $\left(x_{n}\right)$ be a sequence such that $x_{n} \stackrel{b}{\rightarrow} x$ for some $x \in X$. By the forward compactness, the sequence $\left(x_{n}\right)$ has a forward convergent subsequence, say $x_{n_{k}} \stackrel{f}{\rightarrow} y$ as $j \rightarrow \infty$. Then $x=y$, by Lemma 2.2 .

Suppose $\left(x_{n}\right)$ is not forward convergent to $x$. Then there exists a $c \in E$ with $\theta \ll c$ and a subsequence $\left(x_{n_{k}}\right)$ with $c-d\left(x, x_{n_{k}}\right) \notin \stackrel{\circ}{P}$. But this subsequence has a subsequence $x_{n_{k_{j}}}$ forward converging to $x$, so there exists $J \in \mathbb{N}$ such that for all $j \geq J$ one has $d\left(x, x_{n_{k_{j}}}\right) \ll c$, i.e., $c-$ $d\left(x, x_{n_{k_{j}}}\right) \in \stackrel{\circ}{P}$ which is a contradiction. So $x_{n} \stackrel{f}{\rightarrow} x$.

Definition 2.4. A sequence of functions $\left(f_{n}\right)$ from a quasi cone metric space $\left(X, d_{X}\right)$ to a quasi cone metric space $\left(Y, d_{Y}\right)$ is said to be forward convergent (resp. backward convergent) uniformly to $f$ if for $c^{\prime} \in E^{\prime}$ with $\theta^{\prime} \ll c^{\prime}$ and for all $x \in X$ there exists a positive integer $m$ such that

$$
d_{Y}\left(f(x), f_{n}(x)\right) \ll c^{\prime},\left(\operatorname{resp} . d_{Y}\left(f_{n}(x), f(x)\right) \ll c^{\prime}\right), \forall x \in X \text { and } n \geq m .
$$

Note that, here we have taken $d_{Y}$ as a quasi cone metric from $Y \times Y$ to a real Banach space $E^{\prime}$ with a cone $P^{\prime} \subset E^{\prime}$ and $\theta^{\prime}$ is zero in $E^{\prime}$.

Example 2.5. Let $X=[0,1], E=\mathbb{R}^{2}, P=\{(x, y) \in E: x, y \geq 0\}$ and $q: X \times X \rightarrow E$ defined by

$$
q(x, y)= \begin{cases}(x-y, \alpha(x-y)), & x \geq y \\ (\alpha, 1), & x<y\end{cases}
$$

then $q$ is a quasi cone metric on $X$.

(i) Consider the sequence $\left(x_{n}\right)=\left(\frac{1}{n}\right)$, then $x_{n} \stackrel{b}{\rightarrow} 0$ due to

$$
q\left(x_{n}, 0\right)=\left(\frac{1}{n}, \frac{\alpha}{n}\right) \rightarrow 0, \text { as } n \rightarrow \infty .
$$

On the other hand, the sequence $\left(x_{n}\right)$ does not forward converge to 0.

(ii) Consider the sequence $\left(x_{n}\right)=\left(1-\frac{1}{n}\right)$ then $x_{n} \stackrel{f}{\rightarrow} 1$ due to

$$
q\left(1, x_{n}\right)=\left(\frac{1}{n}, \frac{\alpha}{n}\right) \rightarrow 0, \text { as } n \rightarrow \infty .
$$

On the other hand, the sequence $\left(x_{n}\right)$ does not backward converge to 1 .

Theorem 2.6. If $\left(f_{n}\right)$ is a sequence of $f f$-continuous functions from a quasi cone metric space $\left(X, d_{X}\right)$ to quasi cone metric space $\left(Y, d_{Y}\right)$ with forward convergence equivalent to backward convergence and $\left(f_{n}\right)$ forward converges uniformly to a function $f$ then $f$ is $f f$-continuous.

Proof. Let $c^{\prime} \in E^{\prime}$ with $\theta^{\prime} \ll c^{\prime}$ and $\left(x_{n}\right)$ be any forward convergent sequence in $X$ such that $x_{n} \stackrel{f}{\rightarrow} x_{0}$. Since $f_{n} \stackrel{f}{\rightarrow} f$ uniformly, there exists a positive integer $N$ such that

$$
d_{Y}\left(f(x), f_{n}(x)\right) \ll \frac{c^{\prime}}{3}, \quad \forall n \geq N \text { and } x \in X .
$$

In particular, for $n=n_{1}$

$$
d_{Y}\left(f(x), f_{n_{1}}(x)\right) \ll \frac{c^{\prime}}{3} .
$$


Since forward convergence is equivalent to backward convergence, therefore

$$
d_{Y}\left(f_{n_{1}}(x), f(x)\right) \ll \frac{c^{\prime}}{3} .
$$

Furthermore, $\left(f_{n}\right)$ is given to be a sequence of $f f$-continuous functions, therefore

$$
d_{Y}\left(f_{n_{1}}\left(x_{0}\right), f_{n_{1}}\left(x_{n}\right)\right) \ll \frac{c^{\prime}}{3} .
$$

Also from (2.1)

$$
d_{Y}\left(f\left(x_{0}\right), f_{n_{1}}\left(x_{0}\right)\right) \ll \frac{c^{\prime}}{3}
$$

Thus from 2.2), 2.3), and 2.4

$$
d_{Y}\left(f\left(x_{0}\right), f\left(x_{n}\right)\right) \leq d_{Y}\left(f\left(x_{0}\right), f_{n_{1}}\left(x_{0}\right)\right)+d_{Y}\left(f_{n_{1}}\left(x_{0}\right), f_{n_{1}}\left(x_{n}\right)\right)+d_{Y}\left(f_{n_{1}}\left(x_{n}\right), f\left(x_{n}\right)\right) \ll c^{\prime} .
$$

Thus $f$ transforms forward convergent sequence in $X$ to a forward convergent sequence in $Y$. Hence $f$ is an $f f$-continuous function.

An analogous statement can be written for the other three types of continuity.

Theorem 2.7. The set of all $f f$-continuous functions from quasi cone metric space $\left(X, d_{X}\right)$ to quasi cone metric space $\left(Y, d_{Y}\right)$, with forward convergence equivalent to backward convergence, is a closed subset of all continuous functions, i.e., $\mathbb{C}^{f f}(X, Y)=\overline{\mathbb{C}^{f f}(X, Y)}$ where $\mathbb{C}^{f f}(X, Y)$ is the set of all $f f$ continuous functions from quasi cone metric space $\left(X, d_{X}\right)$ to quasi cone metric space $\left(Y, d_{Y}\right)$ and $\overline{\mathbb{C}^{f f}(X, Y)}$ denotes the closure of $\mathbb{C}^{f f}(X, Y)$.

Proof. Let $f \in \overline{\mathbb{C}^{f f}(X, Y)}$. Then there exists a sequence of points in $\overline{\mathbb{C}^{f f}(X, Y)}$ such that $f_{n} \stackrel{f}{\rightarrow} f$ as $n \rightarrow \infty$. Let $c^{\prime} \in E^{\prime}$ with $\theta^{\prime} \ll c^{\prime}$ and $\left(x_{n}\right)$ be any forward convergent sequence in $X$ such that $x_{n} \stackrel{f}{\rightarrow} x_{0}$ as $n \rightarrow \infty$.

Since $f_{n} \stackrel{f}{\rightarrow} f$ uniformly, there exists a positive integer $N$ such that

$$
d_{Y}\left(f(x), f_{n}(x)\right) \ll \frac{c^{\prime}}{3} \forall n \geq N \text { and } x \in X .
$$

In particular, for $n=n_{1}$

$$
d_{Y}\left(f(x), f_{n_{1}}(x)\right) \ll \frac{c^{\prime}}{3} .
$$

Since forward convergence is equivalent to backward convergence, therefore

$$
d_{Y}\left(f_{n_{1}}(x), f(x)\right) \ll \frac{c^{\prime}}{3} .
$$

Furthermore, $\left(f_{n}\right)$ is given to be a sequence of $f f$-continuous functions, therefore

$$
d_{Y}\left(f_{n_{1}}\left(x_{0}\right), f_{n_{1}}\left(x_{n}\right)\right) \ll \frac{c^{\prime}}{3} .
$$

Also, from 2.5

$$
d_{Y}\left(f\left(x_{0}\right), f_{n_{1}}\left(x_{0}\right)\right) \ll \frac{c^{\prime}}{3}
$$

Thus from (2.6), 2.7), and (2.8)

$$
d_{Y}\left(f\left(x_{0}\right), f\left(x_{n}\right)\right) \leq d_{Y}\left(f\left(x_{0}\right), f_{n_{1}}\left(x_{0}\right)\right)+d_{Y}\left(f_{n_{1}}\left(x_{0}\right), f_{n_{1}}\left(x_{n}\right)\right)+d_{Y}\left(f_{n_{1}}\left(x_{n}\right), f\left(x_{n}\right)\right) \ll c^{\prime} .
$$

Hence $f$ is $f f$-continuous function. So $f \in \mathbb{C}^{f f}(X, Y)$ which completes the proof of the theorem. 
Corollary 2.8. The set of all $f f$-continuous functions from a quasi cone metric space $\left(X, d_{X}\right)$ to a quasi cone metric space $\left(Y, d_{Y}\right)$, with forward convergence equivalent to backward convergence, is a complete subspace of the space of all continuous functions.

Theorem 2.9. An $f f$-continuous image of a forward sequentially compact subset of a quasi cone metric space $\left(X, d_{X}\right)$ is forward sequentially compact.

Proof. Let $\left(X, d_{X}\right)$ and $\left(Y, d_{Y}\right)$ be quasi cone metric spaces. Let $f: X \rightarrow Y$ be $f f$-continuous function and $A \subset X$ be forward sequentially compact. Let $\left(y_{n}\right)$ be a sequence in $f(A)$. Then we can write $y_{n}=f\left(x_{n}\right)$ where $x_{n} \in X$ for each $n \in \mathbb{N}$. Since $A$ is forward sequentially compact, there exists a forward convergent subsequence $\left(x_{n_{k}}\right)$ of $\left(x_{n}\right)$. Again it is given that $f$ is $f f$-continuous, this implies that $f\left(x_{n_{k}}\right)$ is forward convergent subsequence of $f\left(x_{n}\right)$. Hence $f(A)$ is forward sequentially compact.

Example 2.10. Let $X=[0,1], E=\mathbb{R}^{2}, P=\{(x, y) \in E: x, y \geq 0\}$ and $q: X \times X \rightarrow E$ defined by

$$
q(x, y)= \begin{cases}(x-y, \alpha(x-y)), & x \geq y \\ (\alpha, 1), & x<y .\end{cases}
$$

Clearly, $q$ is a quasi cone metric on $X$. We define a mapping $f: X \rightarrow X$ by $f(x)=x$. It is easy to see that $X$ is forward sequentially compact and $f$ is an $f f$-continuous function. Clearly, the image space of $f$, i.e., $f(X)$ is also forward sequentially compact.

Corollary 2.11. An fb-continuous image of a forward sequentially compact subset of a quasi cone metric space $\left(X, d_{X}\right)$, with backward convergence equivalent to forward convergence in the image space of $\left(X, d_{X}\right)$, is forward sequentially compact.

An analogous statement can be written for the other types of continuity.

Recalling the concepts of $G$-sequential closure, $G$-sequential accumulation point, and $G$-sequentially compactness given in [5] suggests us to give the following definitions in a quasi-cone metric space $(X, d)$ (see also [6] and [19]).

Definition 2.12. A point $x \in X$ is called forward (resp. backward) sequential accumulation point of $F \subset X$ if there is a sequence $\left(x_{n}\right)$ of points in $F-\{x\}$ such that $x_{n} \stackrel{f}{\rightarrow} x$ (Resp. $x_{n} \stackrel{b}{\rightarrow} x$ ).

Definition 2.13. A subset $F$ of $X$ is called forward (resp. backward) sequentially countably compact if any infinite subset of $F$ has at least one forward (resp. backward) sequential accumulation point in $F$.

Definition 2.14. Let $F \subset X$ and $x \in X$. Then $x$ is in the forward (resp. backward) sequential closure of $F$ if there is a sequence $\left(x_{n}\right)$ of points in $F$ such that $x_{n} \stackrel{f}{\rightarrow} x$ (resp. $x_{n} \stackrel{f}{\rightarrow} x$ ). We denote the forward (resp. backward) sequential closure of $F$ by $\bar{F}^{f}$ (resp. $\bar{F}^{b}$ ). We say that a set is forward (resp. backward) sequentially closed if it contains all of the points in its forward (resp. backward) sequential closure.

Theorem 2.15. A forward sequential compact subset of a quasi cone metric space $(X, d)$ is forward sequentially closed.

Proof. Let $F$ be any forward sequential compact subset of a quasi cone metric space $(X, d)$. Take any $x \in \bar{F}^{f}$. Then there exists a sequence $\left(x_{n}\right)$ of points in $F$ such that $x_{n} \stackrel{f}{\rightarrow} x$. Now since $F$ is forward sequentially compact, there exists a subsequence $\left(x_{n_{k}}\right)$ of $\left(x_{n}\right)$ such that $x_{n_{k}} \stackrel{f}{\rightarrow} x_{0} \in F$. Thus $x_{0}=x \in F$. Therefore, $F$ is sequentially closed in $X$. 
Example 2.16. We consider the same quasi cone metric space as discussed in the Example 2.10. Clearly, the set $A=\left[\frac{1}{2}, 1\right]$ is forward sequentially compact. Consider the sequence $x_{n}=\left(1-\frac{1}{2 n}\right)$ in $A$. Then $\left(x_{n}\right)$ forward converges to $1 \in A$ for

$$
q\left(1, x_{n}\right)=\left(\frac{1}{2 n}, \frac{\alpha}{2 n}\right) \rightarrow 0, \text { as } n \rightarrow \infty .
$$

Hence $A$ is forward sequentially closed.

Theorem 2.17. A subset $F$ of a quasi cone metric space $(X, d)$ is forward sequentially compact if and only if it is forward sequentially countably compact.

Proof. Let $F$ be any forward sequentially compact subset of a quasi cone metric space $(X, d)$ and $B$ be an infinite subset of $F$. Let $\left(x_{n}\right)$ be any sequence of distinct points in $B$. Since $F$ is forward sequentially compact, this sequence $\left(x_{n}\right)$ has a forward convergent subsequence $\left(x_{n_{k}}\right)$ whose limit is $x$, say. Then $x$ is the limit point of $B$. This proves the first part.

Conversely, Suppose that $F$ is forward sequentially countably compact subset of $X$. Let $\left(x_{n}\right)$ be any sequence of points $F$. Let $A=\left\{x_{n}: n \in \mathbb{N}\right\}$. If $A$ is finite then we have nothing to prove. Let $A$ be infinite. Now since $F$ is forward sequentially countably compact, $A$ has a forward sequential accumulation point $x$ in $F$. Now it is not difficult to extract a subsequence $\left(x_{n_{k}}\right)$ of $\left(x_{n}\right)$ such that $x_{n_{k}} \stackrel{f}{\rightarrow} x \in F$. This completes the proof.

An analogous statement can be written for backward case.

Theorem 2.18. Any forward sequentially closed subset of a forward sequentially compact subset of a quasi cone metric space $(X, d)$ is forward sequentially compact.

Proof. Let $A$ be any forward sequentially compact subset of $X$ and $B$ be a forward sequentially closed subset of $A$. Let $\mathbf{x}=\left(x_{n}\right)$ be any sequence of points in $B$. Then $\mathbf{x}=\left(x_{n}\right)$ is a sequence of points in $A$. Since $A$ is forward sequentially compact, there exists a forward convergent subsequence $\left(x_{n_{k}}\right)$ of the sequence $x$. Since $B$ is forward sequentially closed, any sequence $\mathbf{x}=\left(x_{n}\right)$ of points in $B$ has forward convergent subsequence in $B$. Hence the result follows.

Example 2.19. The result is very clear from the previous examples 2.10 and 2.16. We consider the same quasi cone metric space wherein $A=\left[\frac{1}{2}, 1\right]$ is a forward sequentially closed subset of forward sequentially compact set $X=[0,1]$. Now it is easy to see that the set $A=\left[\frac{1}{2}, 1\right]$ is forward sequentially compact.

Corollary 2.20. Any backward sequentially closed subset of a backward sequentially compact subset of a quasi cone metric space $(X, d)$ is backward sequentially compact.

Theorem 2.21. An ff-continuous image of a forward sequentially countably compact set is forward sequentially countably compact.

Proof. Let $\left(X, d_{X}\right)$ and $\left(Y, d_{Y}\right)$ be quasi cone metric spaces. Let $f: X \rightarrow Y$ be an $f f$-continuous mapping. Let $A \subset X$ be forward sequentially countably compact. Let $B$ be an infinite subset of $f(A)$, so that $B$ contains a set $\left\{b_{n}: n \in \mathbb{N}\right\}$.

Then there exists a set $\left\{a_{n}: n \in \mathbb{N}\right\} \subset A$ such that $f\left(a_{n}\right)=b_{n}$ for every $n \in \mathbb{N}$. Now $A$ is forward sequentially countably compact, therefore every infinite subset of $A$ contains forward sequential accumulation point in $A$.

$\Rightarrow\left\{a_{n}: n \in \mathbb{N}\right\}$ has a forward sequential accumulation point in $A$, say $a_{0}$. 


$$
\Rightarrow a_{n} \stackrel{f}{\rightarrow} a_{0} \in A \text {. }
$$

Since $f$ is $f f$-continuous function, so $a_{n} \stackrel{f}{\rightarrow} a_{0} \in A$ implies $f\left(a_{n}\right) \stackrel{f}{\rightarrow} f\left(a_{0}\right) \in f(A)$. That is, $b_{n} \stackrel{f}{\rightarrow} f\left(a_{0}\right) \in f(A)$. Thus $f\left(a_{0}\right)$ is a forward sequential accumulation point in $f(A)$. This proves the result.

Lemma 2.22. A quasi cone metric space is forward complete if every forward Cauchy sequence has a forward convergent subsequence.

Proof. Let $(X, d)$ be a quasi cone metric space and $\left(x_{n}\right)_{n \in \mathbb{N}}$ be a forward Cauchy sequence in $(X, d)$ with subsequence $\left(x_{n_{k}}\right)$ forward converging to $x \in X$. Let $c \in E$ with $\theta \ll c$ so that $d\left(x_{n}, x_{m}\right) \ll \frac{c}{2}$ for all $m \geq n \geq N \in \mathbb{N}$. Choose $k \in \mathbb{N}$ so that $n_{k} \geq N$ and $d\left(x, x_{n_{k}}\right)<<\frac{c}{2}$. Then for $n \geq n_{k} \geq N$

$$
d\left(x, x_{n}\right) \leq d\left(x, x_{n_{k}}\right)+d\left(x_{n_{k}}, x_{n}\right) \ll \frac{c}{2}+\frac{c}{2}=c .
$$

This implies $x_{n} \stackrel{f}{\rightarrow} x$ as $n \rightarrow \infty$. Therefore, $X$ is forward complete.

We introduce some further notations: $Y^{X}$ denotes the space of functions from $X$ to $Y$. The uniform metric on $Y^{X}$ is

$$
\rho(f, g)=\sup \left\{\overline{d_{Y}}(f(x), g(x)): x \in X\right\},
$$

where $\overline{d_{Y}}(f(x), g(x))=\min \left\{d_{Y}((f(x), g(x)), 1)\right\}$ and $d_{Y}$ is a quasi cone metric associated with $Y$.

Theorem 2.23. Let $\left(X, d_{X}\right)$ and $\left(Y, d_{Y}\right)$ be quasi cone metric spaces. If $Y$ is forward complete, then $Y^{X}$ is also forward complete.

Proof. Let $\left(f_{n}\right)_{n \in \mathbb{N}}$ be forward Cauchy sequence in $Y^{X}$. By definition, for $c^{\prime} \in E^{\prime}$ with $\theta^{\prime} \ll c^{\prime}$ there exists $N \in \mathbb{N}$ such that for $m \geq n \geq N$,

$$
\rho\left(f_{n}, f_{m}\right) \ll c^{\prime} .
$$

For $x \in X$, clearly, $f_{n}(x)$ is a forward Cauchy sequence in $Y$. Since $Y$ is forward complete, so $f_{n}(x)$ is forward convergent, say $f_{n}(x) \stackrel{f}{\rightarrow} f(x)$. Thus there exists $N \in \mathbb{N}$ such that $n \geq N$ implies that

$$
d_{Y}\left(f(x), f_{n}(x)\right) \ll c^{\prime}, c^{\prime} \in E^{\prime} \text { with } \theta^{\prime} \ll c^{\prime} .
$$

Taking supremum on $x \in X$, we obtain $f_{n} \stackrel{f}{\rightarrow} f$ in uniform metric $\rho$.

Theorem 2.24. Let $\mathbb{F} \subset Y^{X}$ be a family of $f f$-continuous functions from quasi cone metric space $\left(X, d_{X}\right)$ to quasi cone metric space $\left(Y, d_{Y}\right)$. Let $Y$ be forward complete and forward convergence implies backward convergence in $Y$. Then $\mathbb{F}$ is forward complete.

Proof. Let $f_{n} \subset \mathbb{F}$ such that $f_{n} \stackrel{f}{\rightarrow} f$. Since $Y$ is forward complete, so $Y^{X}$ is also forward complete. Also $\mathbb{F} \subset Y^{X}$, so it is sufficient to show that $f \in \mathbb{F}$.

Now for $x, y \in X, c \in E$ with $\theta \ll c$, there exists $c^{\prime} \in E^{\prime}$ with $\theta^{\prime} \ll c^{\prime}$ such that $d_{X}(x, y) \ll c$ implies

$$
d_{Y}\left(f_{n}(x), f_{n}(y)\right) \ll \frac{c^{\prime}}{3} .
$$

Also there is $N \in \mathbb{N}$ such that

$$
d_{Y}\left(f(x), f_{n}(x)\right) \ll \frac{c^{\prime}}{3}, \quad \forall n \geq N .
$$


Now, forward convergence implies backward convergence, therefore,

$$
d_{Y}\left(f_{n}(y), f(y)\right) \ll \frac{c^{\prime}}{3}, \forall n \geq N
$$

Therefore, $c \in E$ with $\theta \ll c$ and $d_{X}(x, y) \ll c$, we have

$$
d_{Y}(f(x), f(y)) \leq d_{Y}\left(f(x), f_{n}(x)\right)+d_{Y}\left(f_{n}(x), f_{n}(y)\right)+d_{Y}\left(f_{n}(y), f(y)\right) \ll \frac{c^{\prime}}{3}+\frac{c^{\prime}}{3}+\frac{c^{\prime}}{3}=c^{\prime} .
$$

Therefore, $f$ is $f f$-continuous and so $f \in \mathbb{F}$. Thus the result follows.

\section{References}

[1] M. Abbas, G. Jungck, Common fixed point results for noncommuting mappings without continuity in cone metric spaces, J. Math. Anal. Appl., 341 (2008), 416-420. 1

[2] M. Abbas, B. E. Rhoades, Fixed and periodic point results in cone metric spaces, Appl. Math. Lett., 22 (2009), 511-515. 1

[3] T. Abdeljawad, E. Karapinar, Quasicone metric spaces and generalizations of Caristi Kirk's theorem, Fixed Point Theory Appl., 2009 (2009), 9 pages. 1, 1.3

[4] G. E. Albert, A note on quasi-metric spaces, Bull. Amer. Math. Soc., 47 (1941), 479-482. 1

[5] H. Çakallı, Sequential definitions of compactness, Appl. Math. Lett., 21 (2008), 594-598. 2

[6] H. Çakallı, On G-continuity, Comput. Math. Appl., 61 (2011), 313-318. 2

[7] H. Çakallı, Half quasi Cauchy sequences, arXiv preprint, (2012). 1

[8] H. Çakallı, Upward and downward statistical continuities, Filomat, 29 (2015), 2265-2273.

[9] H. Çakall, On variations of quasi-Cauchy sequences in cone metric spaces, Filomat, 30 (2016), 603-610.

[10] H. Çakallı, A. Sönmez, Slowly oscillating continuity in abstract metric spaces, Filomat, 27 (2013), 925-930.

[11] K. P. Chi, T. Van An, Dugundji's theorem for cone metric spaces, Appl. Math. Lett., 24 (2011), 387-390. 1

[12] J. Collins, J. Zimmer, An asymmetric Arzelá-Ascoli theorem, Topology Appl., 154 (2007), $2312-2322$. 1

[13] R. Engelking, General topology, Translated from the Polish by the author, Second edition, Sigma Series in Pure Mathematics, Heldermann Verlag, Berlin, (1989). 1

[14] L. G. Huang, X. Zhang, Cone metric spaces and fixed point theorems of contractive mappings, J. Math. Anal. Appl., 332 (2007), 1468-1476. 1, 1.2

[15] M. Khani, M. Pourmahdian, On the metrizability of cone metric spaces, Topology Appl., 158 (2011), 190-193. 1

[16] H.-P. A. Künzi, A note on sequentially compact quasipseudometric spaces, Monatsh. Math., 95 (1983), 219-220. 1

[17] S. D. Lin, A common fixed point theorem in abstract spaces, Indian J. Pure Appl. Math., 18 (1987), 685-690. 1

[18] A. C. G. Mennucci, On asymmetric distances, Anal. Geom. Metr. Spaces, 1 (2013), 200-231. 1

[19] O. Mucuk, T. Şahan, On G-sequential continuity, Filomat, 28 (2014), 1181-1189. 2

[20] S. Radenović, Z. Kadelburg, Quasi-contractions on symmetric and cone symmetric spaces, Banach J. Math. Anal., 5 (2011), 38-50 1.10

[21] Sh. Rezapour, R. Hamlbarani, Some notes on the paper: "Cone metric spaces and fixed point theorems of contractive mappings", J. Math. Anal. Appl., 345 (2008), 719-724. 1

[22] B. Rzepecki, On fixed point theorems of Maia type, Publ. Inst. Math. (Beograd) (N.S.), 28 (1980), 179-186. 1

[23] F. Shadad, M. S. Md. Noorani, Fixed point results in quasi-cone metric spaces, Abstr. Appl. Anal., 2013 (2013), 7 pages. 1.5, 1.7, 1, 1.9

[24] A. Sönmez, On paracompactness in cone metric spaces, Appl. Math. Lett., 23 (2010), 494-497. 1]

[25] A. Sönmez, Fixed point theorems in partial cone metric spaces, arXiv preprint, (2011). 1 .

[26] D. Turkoglu, M. Abuloha, Cone metric spaces and fixed point theorems in diametrically contractive mappings, Acta Math. Sin. (Engl. Ser.), 26 (2010), 489-496. 1 
[27] J. S. Vandergraft, Newton's method for convex operators in partially ordered spaces, SIAM J. Numer. Anal., 4 (1967), 406-432. 1

[28] W. A. Wilson, On Semi-Metric Spaces, Amer. J. Math., 53 (1931), 361-373. 1

[29] T. Yaying, B. Hazarika, Some new types of continuity in asymmetric metric space, Contem. Anal. Appl. Math., (Accepted). 1 